\title{
Stress Induces a Shift Towards Striatum-Dependent Stimulus-Response Learning via the Mineralocorticoid Receptor
}

\author{
Susanne Vogel ${ }^{*, 1,2}$, Floris Klumpers ${ }^{1,2}$, Tobias Navarro Schröder ${ }^{3}$, Krista T Oplaat ${ }^{1,2}$, Harm J Krugers ${ }^{4}$, \\ Melly S Oitzl ${ }^{4}$, Marian Joëls ${ }^{5}$, Christian F Doeller ${ }^{3}$ and Guillén Fernández ${ }^{1,2}$ \\ 'Radboud University Medical Center, Donders Institute for Brain, Cognition and Behavior, Nijmegen, The Netherlands; ${ }^{2}$ Radboud University Medical \\ Center, Department of Cognitive Neuroscience, Nijmegen, The Netherlands; ${ }^{3}$ Radboud University, Donders Institute for Brain, Cognition and \\ Behavior, Nijmegen, The Netherlands; ${ }^{4}$ Faculty of Science, Swammerdam Institute for Life Sciences, University of Amsterdam, Amsterdam, The \\ Netherlands; ${ }^{5}$ Rudolf Magnus Institute of Neuroscience, Department of Translational Neuroscience, Utrecht, The Netherlands
}

\begin{abstract}
Stress is assumed to cause a shift from flexible 'cognitive' memory to more rigid 'habit' memory. In the spatial memory domain, stress impairs place learning depending on the hippocampus whereas stimulus-response learning based on the striatum appears to be improved. While the neural basis of this shift is still unclear, previous evidence in rodents points towards cortisol interacting with the mineralocorticoid receptor (MR) to affect amygdala functioning. The amygdala is in turn assumed to orchestrate the stress-induced shift in memory processing. However, an integrative study testing these mechanisms in humans is lacking. Therefore, we combined functional neuroimaging of a spatial memory task, stress-induction, and administration of an MR-antagonist in a full-factorial, randomized, placebo-controlled between-subjects design in $10 \mathrm{I}$ healthy males. We demonstrate that stress-induced increases in cortisol lead to enhanced stimulusresponse learning, accompanied by increased amygdala activity and connectivity to the striatum. Importantly, this shift was prevented by an acute administration of the MR-antagonist spironolactone. Our findings support a model in which the MR and the amygdala play an important role in the stress-induced shift towards habit memory systems, revealing a fundamental mechanism of adaptively allocating neural resources that may have implications for stress-related mental disorders.

Neuropsychopharmacology (2017) 42, I262-127I; doi:I0.1038/npp.2016.262; published online 2I December 2016
\end{abstract}

\section{INTRODUCTION}

Encountering stressful events triggers a well-described cascade of neural changes (Joëls and Baram, 2009), which ultimately also affects memory. Whereas earlier studies focused on stress effects on memory quantity, recent studies illustrated that stress also changes memory quality by shifting the balance of systems underlying learning (Hermans et al, 2014; Schwabe and Wolf, 2013b) and retrieval (Elliott and Packard, 2008). Accordingly, memory formation under stress appears to be dominated by inflexible, 'habitual' forms of learning such as stimulusresponse learning based on the striatum. In contrast, the contribution of more 'cognitive' learning centered at the hippocampus appears reduced under stress (Packard and

*Correspondence: Dr S Vogel, Institute for Psychology, Department of Cognitive Psychology, University of Hamburg, Von-Melle-Park 5, Hamburg 20146, Germany, Tel: +49 4042838 5892, Fax: +49 40 42838-4373,

E-mail: Susanne.vogel@uni-hamburg.de

Received 10 December 20 I5; revised 2 I September 20 I6; accepted I4 November 2016; accepted article preview online 23 November 2016
Teather, 1998; Packard and Wingard, 2004; Wingard and Packard, 2008).

This shift towards habit memory is often assumed to be adaptive by enabling learning and recall of simple stimulusresponse associations in the face of limited cognitive resources and high external demands (Schwabe and Wolf, 2013b). Nonetheless, the shift might prove relevant for several psychiatric disorders involving well-learned but maladaptive responses to salient cues. For instance, reflexive, often generalized stimulus-fear associations concurrent with impaired hippocampal memory functioning might underlie post-traumatic stress disorder (Acheson et al, 2012). Also patients suffering from addiction or obsessive compulsions might be prone to relapse when maladaptive habits are retrieved under stress (Herman and Polivy, 1975; Weiss et al, 2001).

The stress-induced shift was first described for spatial memory. Under stress, rodents and humans preferentially use simple responses related to salient landmarks that are encoded egocentrically and depend on the striatum (Elliott and Packard, 2008; Kim et al, 2001; Packard and Teather, 1998; Packard and Wingard, 2004; Schwabe et al, 2007; Schwabe et al, 2010; Wingard and Packard, 2008). In 
contrast, stress impairs more complex, allocentric, spatial representations that are based on the hippocampus (Morris et $a l, 1982)$ and allow flexible navigation. Whereas the neural mechanism underlying this stress-induced shift is still unclear in humans, in rodents the shift appears to be mediated by amygdala processing that enhances stimulusresponse learning and decreases place learning (Packard and Teather, 1998; Packard and Wingard, 2004). Other studies in rodents suggested that this shift depends on corticosteroids binding to the mineralocorticoid receptor (MR) (Schwabe et al, 2010) which can rapidly activate the amygdala in-vitro (Karst et al, 2010). In humans, MR-activation under stress was associated with increased functional connectivity between amygdala and striatum in tasks probing emotional face processing and classification learning, possibly contributing to the stress-induced shift (Schwabe et al, 2013a; Vogel et al, 2015b).

Here, we set out to translate these findings to humans and reveal the neural mechanisms underlying a stress-induced shift in human spatial memory. We hypothesized that a stress-induced shift would be mediated by cortisol activating the MR and leading to a dominance of striatal stimulusresponse learning. Furthermore, we expected this shift to be mediated by changes in amygdala activity and connectivity to the striatum. To test these hypotheses, we used a fullfactorial design, investigating the effects of acute stress and MR-blockade on the systems contributing to spatial memory in healthy men.

\section{MATERIALS AND METHODS}

The study was approved by the local ethical committee (NL37819.091.11) and registered in the Dutch (3595) and European trial registry (2011-003493-85). The current study was part of a large-scale study investigating stress-effects depending on MR-availability. Two other data sets acquired in the same participants using independent tasks investigated the role of the MR in emotional face processing (Vogel et al, 2015b) and fear learning (Vogel et al, 2015a). Thus, the overall study set-up was described elsewhere and is only briefly summarized here, focusing on the spatial memory experiment.

\section{Participants}

Healthy right-handed male volunteers $(N=101)$ with normal weight $(18.5 \leqslant$ body mass index $\leqslant 30)$ were included after being screened for the exclusion criteria described in Supplementary Information. All participants provided written informed consent and were financially compensated. Three participants were excluded due to either panic attacks, motion sickness, or the discovery of concurrent participation in another drug study. This resulted in a final number of 98 participants (mean age 21.9 years $(S D=2.9)$ ) not differing in age, body-mass-index, or trait anxiety (Vogel et al, 2015a, 2015b).

\section{General Procedure}

We used a 2-by-2 full-factorial design with the factors stress and MR-blockade. Participants were randomly assigned to one of four experimental groups (control/placebo, stress/ placebo, control/MR-blocked, stress/MR-blocked). While the factor MR-blockade was manipulated in a double-blind fashion, the factor stress was not blinded.

Drug administration and adaptation phase. Testing took place in the afternoon to ensure relatively stable endogenous cortisol levels at baseline. After assessment of baseline cortisol, subjective mood, and vital signs (blood pressure, heart rate) (Supplementary Information), participants were orally administered $400 \mathrm{mg}$ spironolactone (tablets; Teva Pharmachemie, Haarlem, The Netherlands) or placebo. Afterwards, participants practiced the spatial memory task and rested for $80 \mathrm{~min}$ to ensure adaptation to the laboratory and drug absorption. Cortisol and vital signs were measured every $30 \mathrm{~min}$.

Experimental phase. Inside the MRI room, participants underwent a stress induction or a non-stressful control procedure (described below). A short emotional face processing task followed (Vogel et al, 2015b). Approximately $17( \pm 4)$ min after stress induction, participants started with the spatial memory task. After the subsequent fear memory task (Vogel et al, 2015a), participants were debriefed about the stressor and left after a general assessment of well-being.

\section{Stress Induction}

We adapted the socially evaluated cold pressure task (SECPT, Schwabe et al, 2008b) to an MRI-compatible version. Participants were in a supine position on the scanner bench, immersed their right foot into ice water $\left(0-2{ }^{\circ} \mathrm{C}\right)$ up to the ankle, and held it there as long as possible (task stopped after $3 \mathrm{~min}$ ). During foot immersion, participants looked into a camera while being closely observed by two non-supportive experimenters in laboratory coats. Participants performed a difficult mental arithmetic test after the spatial memory task to ensure sustained elevations of stress for the fear task. For the control group, warm water was used $\left(35-37^{\circ} \mathrm{C}\right)$, there was no camera, the experimenter was friendly and casually dressed, and the arithmetic test was simple. To assess whether stress was successfully induced, negative mood, salivary cortisol levels, and vital signs were measured repeatedly and analyzed as described in Supplementary Information.

\section{Spatial Memory Task and Virtual Environment}

We used a spatial memory task based on the Morris water maze (Morris et al, 1982) that allows for distinction between striatal and hippocampal learning systems for stimulusresponse learning and spatial map-based learning, respectively (Doeller and Burgess, 2008a; Doeller et al, 2008b, Figure 1a). UnrealEngine2 Runtime software (Epic Games) was used to present a virtual first-person perspective view of an environment surrounded by a boundary with orientation cues (mountains, clouds, and the sun), and a traffic cone as an intramaze landmark cue. Orientation cues were projected at infinity to provide orientation but no location information within the arena. The landmark cue and the boundary (circular wall) were rotationally symmetric leaving the orientation cues as the sole source of orientation, presumably mediated by the head-direction system (Taube, 1998; Wilton 
a
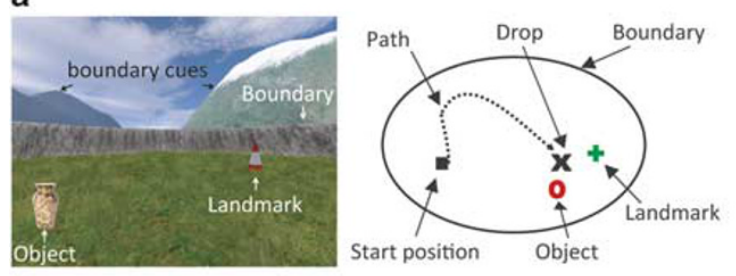

b

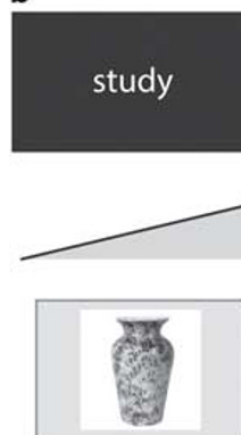

object

2

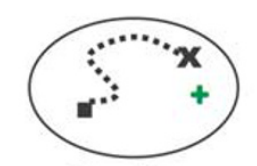

Recall
Feedback

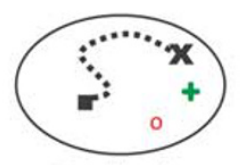

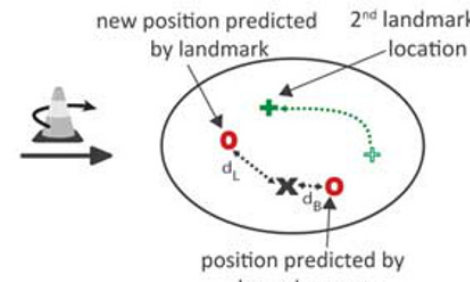

boundary cues

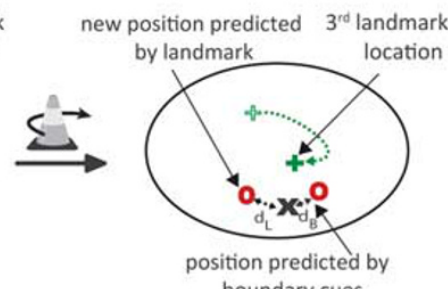

boundary cues
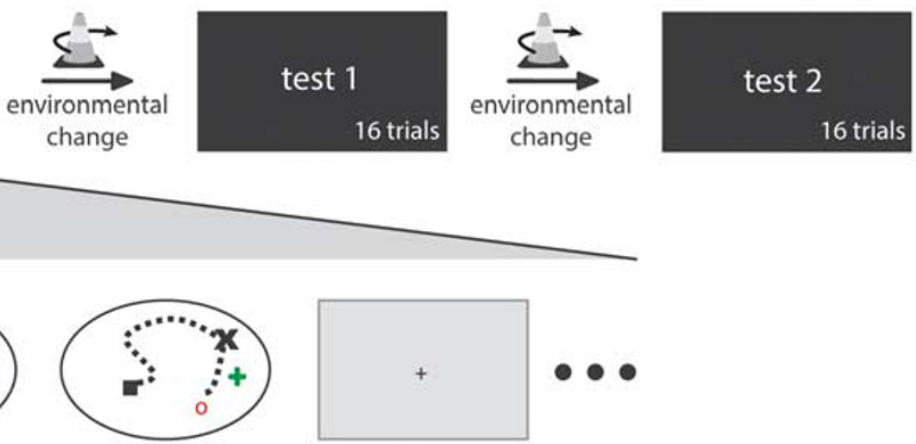

Correct

$\sim 6$

time[s]

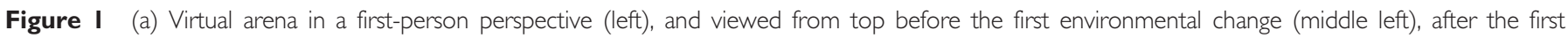

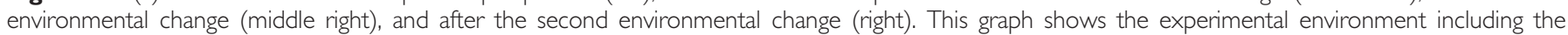

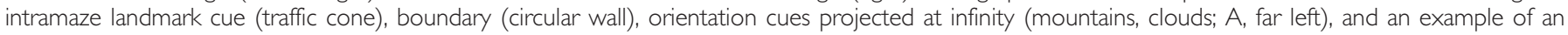

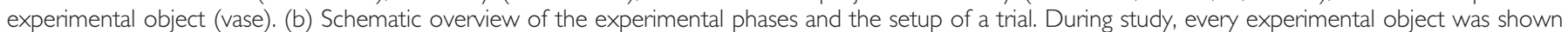

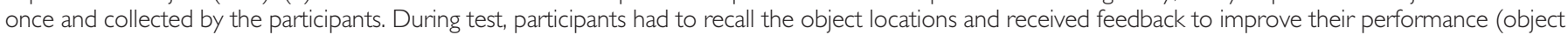

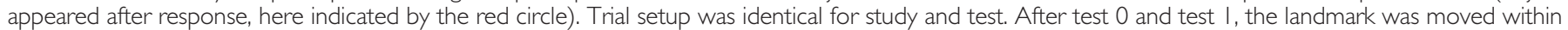

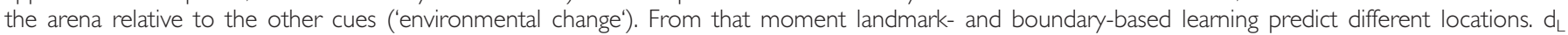

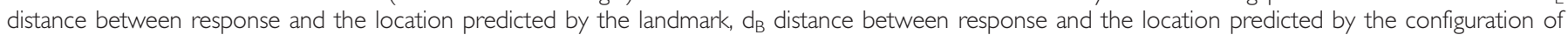
boundary and orientation cues.

et al, 2001). Participants navigated with their right hand operating four buttons to 'move' and 'drop' objects. The viewpoint was $\approx 2 \mathrm{vm}$ above the ground, the arena was $\approx 32 \mathrm{vm}$ in diameter, and the virtual location was recorded every $100 \mathrm{~ms}$.

Practice. The practice environment was used to familiarize participants with the task outside the MRI scanner. Participants saw four everyday objects, once each, at different locations in the arena and were instructed to collect them by 'walking' over them and remember their locations. Subsequently, participants were presented with two trials per object. Trials were separated into mini-blocks, each object occurring once per mini-block. Object order within the miniblocks was semi-random with no object appearing in two successive trials across mini-blocks. Each trial started with an image showing the object to be recalled (2s) after which participants had to 'move' from a random start position to the object's position (recall, Figure 1b). After 'dropping' the object, the object appeared on its correct location (feedback) so that participants could improve their performance on the next trial. Trials were separated by a fixation cross $(4 \mathrm{~s})$.

Study. For the main task in the MRI scanner, four new objects were presented, once each, in an arena with different landmark, boundary, and orientation cues. Participants were again instructed to remember their locations.
Test. After study, participants performed three test blocks (test 0,1 , and 2) with 16 trials each, four per experimental object. Trial timing was similar to the practice task. Critically, without the participants' prior knowledge, the landmark was moved relative to the boundary between test block 0 and 1 and test block 1 and 2 ('environmental changes'), allowing the differentiation of object types from test 1 onwards. Two experimental objects maintained a fixed position relative to the boundary (boundary-based objects), and the two other objects remained in a fixed position relative to the landmark (landmark-based objects). On average, participants took $16 \mathrm{~min}( \pm 1 \mathrm{~min})$ to complete the task.

We recorded reaction time (until object drop), recall error (in $\mathrm{vm}$ ), and the relative influence of boundary or landmark on drop location. As our hypotheses were specifically targeted at the dissociation between spatial map learning and stimulus-response learning, we focused on the 'cue influence' parameter (Doeller et al, 2008b) indicating which environmental cue dominated performance in a given trial. The 'cue influence' parameter was calculated for each trial after the first environmental change occurred (Doeller et al, 2008b). The formula uses the error in the participant's response as distance relative to (i) the position predicted by the landmark cue $\left(\mathrm{d}_{\mathrm{L}}\right)$ and (ii) the position predicted by the boundary cue $\left(d_{B}\right.$, Figure 1$)$. A smaller $d_{L}$ thus indicated a relatively strong influence of the landmark cue on the participant's response, suggesting stimulus-response 
learning. Conversely, a small $\mathrm{d}_{\mathrm{B}}$ indicated a strong influence of the boundary cue, suggesting spatial map-based learning. The ratio between these scores indexes each participant's relative reliance on either boundary or landmark $\left(d_{L} /\right.$ $\left(d_{L}+d_{B}\right)$. The resulting relative influence parameter theoretically varies between 0 (relying exclusively on the landmark) and 1 (relying exclusively on the boundary). After the second environmental change, the incorrect cue (the boundary for landmark-based objects and the landmark for boundarybased objects) predicted two different locations. Consistent with previous work, we used whichever difference to the response location was smaller to calculate the 'cue influence' parameter (Doeller et al, 2008b). A trial of special interest is the first trial after the first environmental change, as it gives an indication of the initial preference for either memory system when participants do not know yet if objects are boundary- or landmark-based. Similarly, earlier human and rodent experiments used this first trial after learning to determine per participant which memory system has been used (Schwabe et al, 2008a).

\section{Magnetic Resonance Imaging and Analysis}

Functional and structural magnetic resonance imaging (MRI) data were acquired on a 1.5T Avanto scanner (Siemens, Germany). Details on acquisition and preprocessing using SPM8 (Wellcome Trust Centre for Neuroimaging, London) can be found in Supplementary Information. To test the main task effects and the sensitivity of brain regions to either the landmark cue or the boundary cue during recall and feedback, we set up a model including regressors for the study phase, object, recall, and feedback, all convolved with the canonical hemodynamic response function (HRF, see Supplementary Information). In line with previous work, the recall regressor was parametrically modulated by the cues' influence on the response (calculated as described above; Doeller et al, 2008b) and the feedback regressor was modulated by the amount of learning (performance increase in the following trial with the same object; Doeller et al, 2008 b, see Supplementary Information). Six realignment parameters were included to account for residual motion. A full-factorial design was employed to test for group differences in brain activity.

A second model was used to investigate brain regions in which activation was associated with the stress-induced cortisol increase. This model contained regressors for object, study, recall, and feedback, the latter two split for boundarybased and landmark-based objects to investigate both memory systems separately. As we did not hypothesize trial-by-trial variation in the effects of cortisol on neural activation, the parametric modulators were omitted from this model for parsimony. All regressors were convolved with the $\mathrm{HRF}$ and six realignment parameters were added to capture residual motion artifacts. Over subjects, we correlated brain activity to the increase in cortisol, calculated as area under the curve with respect to the increase from the beginning of the experimental phase until the end of the spatial memory task (AUCi; Pruessner et al, 2003). A full-factorial design was used to investigate group differences in this correlation, which showed evidence for a stress-induced upregulation of amygdala activity, mediated by cortisol interacting with the MR (see Results).
To test the hypothesis of enhanced amygdala-striatal connectivity under stress, we extracted the time course of amygdala activity using the 'volume of interest' tool as implemented in SPM8. The bilateral seed was functionally defined as the combination of stress-by-MR-blockade interactions on the association with cortisol AUCi in the amygdala. Correlating this time series to activity in the rest of the brain provides information on regions that are supposedly functionally connected with the amygdala. We added the time series to the first level models and accounted for global signal fluctuations by adding two regressors modeling the signal from individually defined white matter and cerebrospinal fluid masks. Again, a full-factorial design was used to test for group differences.

For whole-brain analyses, the significance threshold was set to $p<0.05$ (family-wise error corrected [FWE] at clusterlevel). For our bilateral regions of interest (ROIs, hippocampus, putamen, caudate, amygdala), we implemented small volume correction (SVC), using an initial threshold of $p<0.005$, uncorrected, followed by voxelwise FWEcorrection $(p<0.05)$ for multiple comparisons within ROIs. Bilateral anatomical masks were taken from the Automated Anatomical Labeling (AAL) atlas (Tzourio-Mazoyer et al, 2002) using Wake Forest University PickAtlas 2.4. Given our hypothesis concerning an MR-dependent stress-induced shift, we focus on effects of stress, possibly mediated by MR-activation.

\section{RESULTS}

\section{Stress Measures in the Adaptation Phase}

Negative mood, cortisol levels, heart rate, and blood pressure decreased throughout the adaptation phase indicating successful adaptation to the laboratory environment (all main effects of time $p<0.001)$. MR-blockade led to higher cortisol levels before stress induction (time-by-MR-blockade interaction, $\left.\mathrm{F}_{(1.7,155.4)}=13.33, p<0.001 ; \mathrm{t}_{96}=3.13, p=0.002\right)$ in line with a regulatory role of the MR on cortisol release (de Kloet et al, 2005). Importantly, within both medication groups there was no significant difference between stress and control in any measure prior to stress induction (all $p>0.1$ ).

\section{Stress Measures in the Experimental Phase}

The stress group immersed their foot in water for less time than the control group $\left(\mathrm{F}_{(1,93)}=20.12, p<0.001\right)$, but importantly there was no influence of MR-blockade (main effect or interaction). Stress-related increases in negative mood, cortisol, and, at trend-level, heart rate demonstrated successful stress induction in both medication groups (negative mood: stress main effect $(\mathrm{F}(1,91)=10.91$, $p=0.001)$, time-by-stress interaction $(\mathrm{F}(2.4,218.4)=9.81$, $p<0.001)$; cortisol: stress main effect $(\mathrm{F}(1,92)=13.00$, $p=0.001)$, time-by-stress interaction $(\mathrm{F}(2.5,229.5)=8.93$, $p<0.001)$; heart rate: time-by-stress interaction ( $\mathrm{F}$ $(6.0,525.9)=2.00, p=0.065)$; see Figure 2). Although there was a trend for MR-blockade to reduce stress-induced negative mood (time-by-stress-by-MR-blockade interaction $\mathrm{F}(2.4,218.4)=2.69, p=0.060$, placebo: $\mathrm{F}(2.2,93.5)=10.269$, $p<0.001$, MR-blocked: $p>0.1)$, MR-blockade did not affect stress-induced increases in heart rate or cortisol (all $p>0.4$ ). 

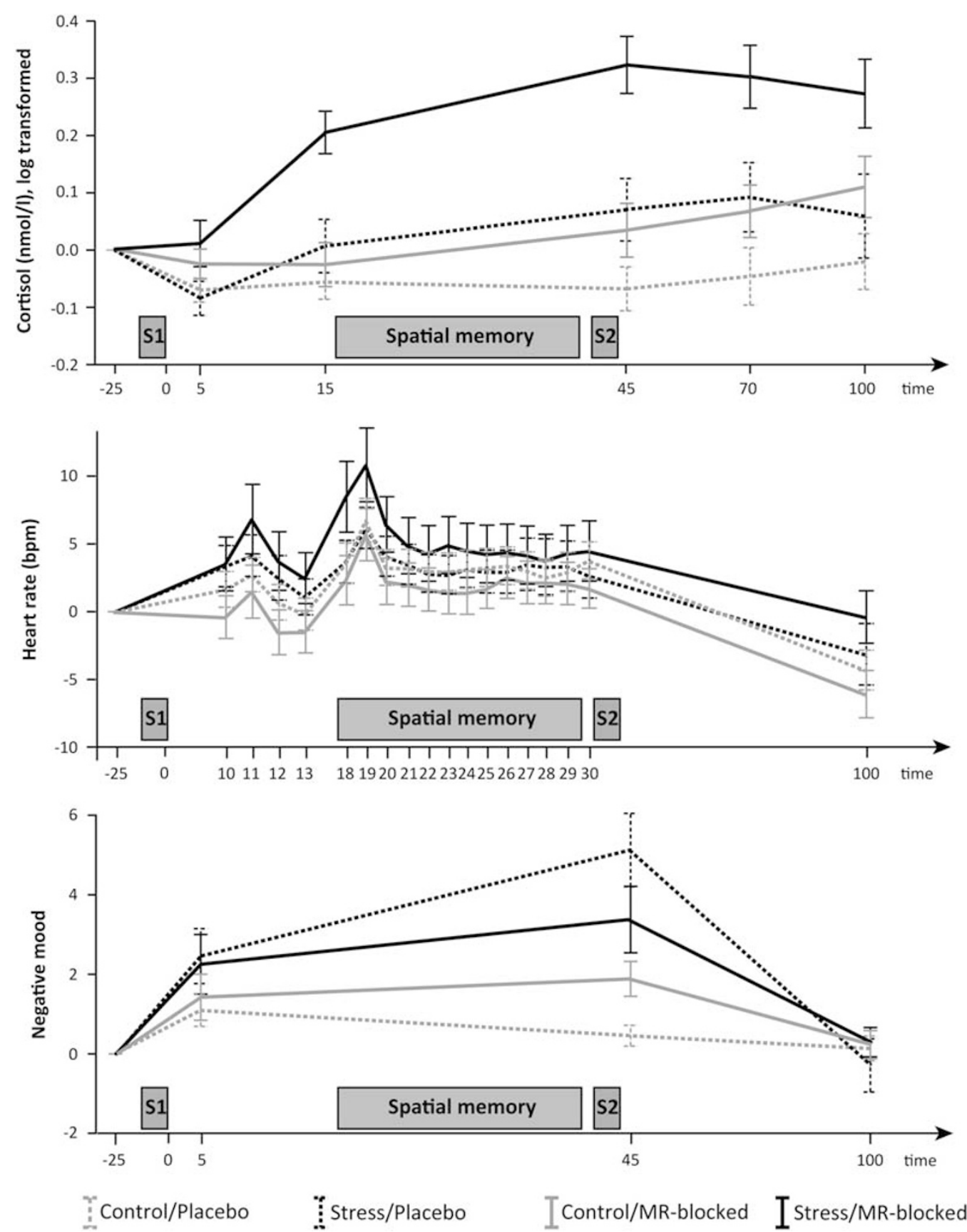

Figure 2 Cortisol levels (top), heart rate (middle), and negative mood (bottom) over the course of the experiment. Participants were randomly assigned to one of four groups: control/placebo (gray dotted lines), stress/placebo (black dotted), control/MR-blocked (grey solid), stress/MR-blocked (black solid). After pill ingestion and habituation to the laboratory environment, participants were brought to the MRI room and underwent either the socially evaluated cold pressure test (SI) or a non-stressful control procedure. After another task probing emotional face processing (Vogel et al, 20 I5b), participants performed the spatial memory task. Stress-related increases in negative mood, cortisol, and, at trend level, heart rate displayed successful stress induction in both drug groups. Time is indicated in minutes after stress induction/control procedure, all measurements are baseline corrected to the last measurement during habituation ( -25 min). Mean values are depicted, error bars represent I SEM; bpm, beats per minute; MR, mineralocorticoid receptor; SI, first stress induction/control procedure (socially evaluated cold pressure task); S2, second stress induction/control procedure (mental arithmetic test) to maintain stress levels for a subsequent task.

Accordingly, cortisol and negative mood levels were elevated in the stress group compared to the control group during the spatial memory task whereas the heart-rate increase wore off rapidly. As expected, MR-blockade led to heightened cortisol levels (MR-blockade main effect: $\mathrm{F}_{(1,92)}=15.01, p<0.001$, time-by-MR-blockade interaction: $\mathrm{F}_{(2.5,229.5)}=6.22, p=0.001$ ) (de Kloet et al, 2005), but did not affect blood pressure or heart rate.

\section{Effects of Stress and MR Blockade on Reaction Times and Recall Performance}

Reaction times were generally shorter for landmark-based objects than boundary-based objects $\left(\mathrm{F}_{(1,87)}=7.676\right.$, $p=0.007$ ), but not affected by stress (all $p>0.4$ ). The MR-blocked group was on average faster than the placebo group (main effect of MR-blockade, $\mathrm{F}_{(1,87)}=4.474$, 
$p=0.037)$, but again, this was independent of stress $(p>0.4)$. Recall errors did not differ between landmark-based and boundary-based objects $(p>0.6)$ and were not affected by stress or MR-blockade (all $p>0.10$ ). To summarize, MRblockade led to faster responses in general, but did not alter response accuracy. Stress, as induced here, did not affect reaction times or recall errors. Thus, a potential shift in spatial memory systems under stress cannot be readily explained by a general performance difference between groups.

\section{The Hippocampal and Striatal Memory Systems are Preferentially Recruited for Boundary and Landmark- Related Objects Respectively}

We next tested whether we could dissociate different learning strategies using the cue influence parameter. In line with previous findings (Doeller et al, 2008b), response locations were influenced by both boundary and landmark cues, and participants learned over time to use the correct cue for each object. Thus, the influence parameter increased for boundary-based objects and decreased for landmarkbased objects indicating a stronger map-based learning for boundary-based objects and stimulus-response learning for landmark-based objects, respectively (main effect of time: $\mathrm{F}(5.2,455.9)=9.83, p<0.001$; object: $\mathrm{F}(1,87)=255.47$, $p<0.001$; time-by-object interaction: $\mathrm{F}(5.2,454.3)=50.29$, $p<0.001$; Figure 3 ). As expected, brain activity during recall tracked the influence of boundary versus landmark cues on replace location (Doeller et al, 2008b). Thus, hippocampal activity was enhanced when the response location was influenced by the boundary cue $\left(\mathrm{p}_{\mathrm{SVC}}=0.021, \mathrm{p}_{\mathrm{SVC}}=0.052\right.$

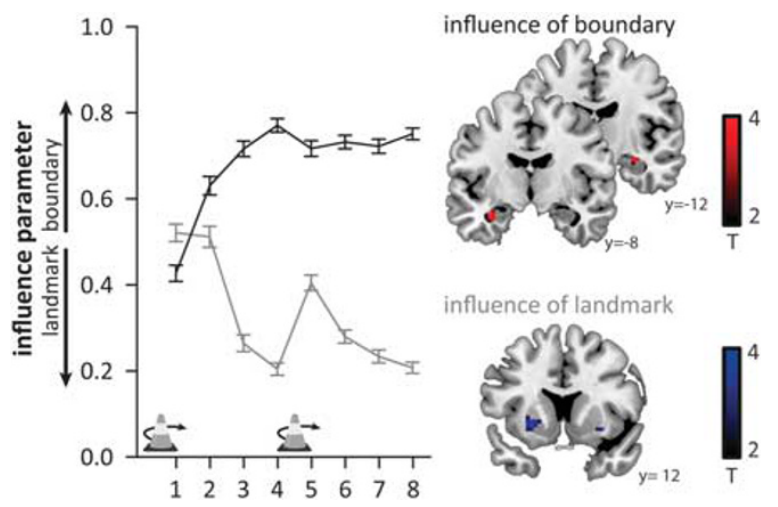

Figure 3 Left: Relative influence of boundary and landmark on the replace location during test I and 2 for objects located relative to the boundary (black) and objects located relative to the landmark (gray). The influence was calculated according to (Doeller et al, 2008b) as dL/(dL+dB), with $\mathrm{dL}$ being the distance of the response from the location predicted by the landmark and $\mathrm{dB}$ being the distance from the location predicted by the boundary. Consequently, the influence varies theoretically between 0 (relying on the landmark) and I (relying on the boundary). Participants learned over time to dissociate landmark-based and boundary-based objects and used the correct cue for each object. Environmental changes are indicated by traffic cone icons with an arrow. Error bars represent I SEM. Right: Activity during recall is modulated by a stronger influence of the boundary in the hippocampus and (at trend level) by a stronger influence of the landmark in the putamen. For illustrative purposes, the images are thresholded at $p<0.005$, uncorrected, and masked for the regions of interest (hippocampus, putamen). (trend-level), Figure 3) and striatal activity was increased (at trend-level) when the response was influenced more by the landmark (putamen: $\mathrm{p}_{\mathrm{SvC}}=0.084$, Figure 3). Furthermore, striatal activity was enhanced when participants received feedback about landmark-based objects which improved their performance (caudate: $\mathrm{p}_{\mathrm{SVC}}=0.010, \mathrm{p}_{\mathrm{SVC}}=0.037$, Supplementary Figure S1).

To summarize, we confirmed that participants used two memory systems during recall and updating. The hippocampal memory system was active when participants used a spatial map-based strategy and relied on the boundary to recall an object's location. In contrast, the striatal memory system was involved when relying on the landmark suggesting stimulus-response learning.

\section{Stress and MR-Blockade Affect the Shift between Memory Systems}

Next, we investigated whether stress and MR-blockade affected the use of these memory systems. In line with our hypothesis of a stress-induced shift, we found a time-bystress-by-MR-blockade interaction on the influence parameter independent of object-type $\left(\mathrm{F}_{(5.2,455.9)}=3.15\right.$, $p=0.007)$. This interaction originated from a significant stress-by-MR-blockade interaction in the first trial after the first environmental change $\left(\mathrm{F}_{(1,92)}=5.91, p=0.017\right)$ but not in later trials. As expected, stress led to a numerically but non-significant increased reliance on the landmark in the placebo group $(p=0.232)$, and the effect was reversed in the MR-blocked groups (stress/MR-blocked vs control/MRblocked: $p=0.023$; stress/MR-blocked $v s$ stress/placebo: $p=0.006$, see Supplementary Figure S2). To account for the large inter-individual differences in the spontaneous use of spatial memory strategies (eg Iaria et al, 2003), we reran the analyses with per participant $z$-transformed influence parameters. These can be interpreted as variation from the within-participant average reliance on boundary or landmark cues. Supporting our previous findings, a repeated measures ANOVA with the within-subject factors trial and object-type and the between-subjects factors stress and MRblockade showed a time-by-stress-by-MR-blockade interaction on the influence parameter $\left(\mathrm{F}_{(5.6,484.3)}=2.602\right.$, $p=0.020)$, originating from the first trial after the first environmental change $\left(\mathrm{F}_{(1,92)}=10.183, p=0.002\right)$. Stress again increased the reliance on the landmark in the placebo groups compared to the control group $(p=0.013$, Figure 4$)$ in the first trial and this effect was reversed in the MR-blocked groups $(p=0.001$; stress/MR-blocked $v s$ stress/ placebo: $p=0.001)$. Finally, this analysis also revealed a main effect of stress $(p=0.017)$, with the stress group showing a stronger influence of the landmark than the control group.

\section{Stress-Induced Cortisol Increases Activate the Amygdala Depending on MR-Availability}

To test how the stress-induced shift might arise on the neural level, we first tested whether striatal or hippocampal engagement during recall or feedback was affected by a stress-by-MR-blockade interaction similar to the behavioral finding. However, no such effects were detected in the striatum, hippocampus, or amygdala (all $\mathrm{p}_{\mathrm{SvC}}>0.4$ ). In a subsequent step, given our specific hypothesis, cortisol-MR 


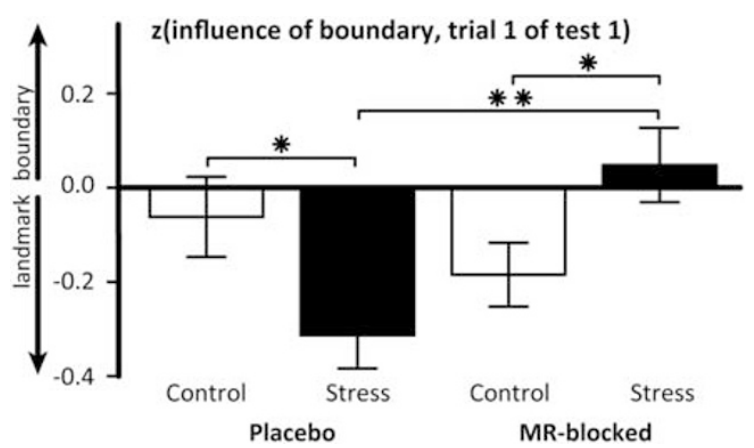

Figure 4 Mean influence of the boundary and the landmark on the first trial in test I across all objects (also shown in Figure 3). The relative use of boundary or landmark in this trial directly after the first environmental change can be interpreted as an indicator for the strategy used to learn the object locations during study and test 0 . This plot shows the within-subject $z$-transformed values, which can be interpreted as variation from the withinparticipant average reliance on boundary or landmark cues over all trials. Mean values are depicted, error bars represent I SEM, MR mineralocorticoid receptor, $* * * 0.01, * p<0.05$.

Table I Stress-by-MR-Availability Interactions on The Correlation Between Cortisol Increase and Amygdala Activity

\begin{tabular}{llllc}
\hline Task phase & Hemisphere & T $_{\text {max }}$ & k & Psvc \\
\hline Recall landmark-based objects & Left & 2.95 & 5 & $0.080^{\#}$ \\
Recall boundary-based objects & Left & 3.15 & 7 & $0.049 *$ \\
& Right & 2.83 & 2 & $0.089^{\#}$ \\
Feedback landmark-based objects & Left & 4.92 & 38 & $<0.00$ I $^{* * *}$ \\
& Right & 4.26 & 40 & $0.002^{* *}$ \\
Feedback boundary-based objects & Left & 3.72 & 36 & $0.012^{*}$ \\
& Right & 4.01 & 34 & $0.005^{* *}$ \\
\hline
\end{tabular}

${ }^{* *} p<0.001,{ }^{* *} p<0.01,{ }^{*} p<0.05,{ }^{*} p<0.10$.

interactions mediate the stress-induced shift, we assessed how inter-individual differences in the cortisol response to stress relate to brain activity. In line with a stress-induced MR-dependent upregulation of amygdala activity, we found stress-by-MR-blockade interactions on the correlation between cortisol increase and amygdala activity during recall and feedback for both object-types (Table 1, Supplementary Figure S2). As expected, participants with higher cortisol increases after stress in the placebo group showed stronger amygdala responses relative to those with smaller cortisol increases (recall boundary-based objects $\mathrm{p}_{\mathrm{SVC}}=0.087$ (trend); feedback landmark-based objects $\mathrm{p}_{\text {SVC }}=0.010, \quad \mathrm{p}_{\mathrm{SVC}}=0.004 ; \quad$ feedback boundary-based objects: $\left.p_{s v C}=0.025\right)$. Crucially, in line with a critical role for the MR in driving this association in the stress group, the correlation was abolished in the stress group where the MR was pharmacologically blocked (no significant voxels).

Perhaps related to the physiological cortisol increase following MR blockade, we found a positive association between cortisol increase and amygdala activity also in the control/MR-blocked group ( $\mathrm{p}_{\mathrm{SVC}}<0.05$ for recall and feedback of both object-types), yet as mentioned above the association was not present in the stress/MR blocked group.

\section{Stress and MR-Blockade Differentially Affect Amygdala Connectivity}

Considering that amygdala activity showed a positive association with the stress-induced cortisol increase in the placebo group, we tested whether the amygdala may drive the stress-induced shift towards enhanced stimulus-response learning via connectivity with the striatum (Schwabe et al, 2013a; Vogel et al, 2015b). Indeed, we found a stress-by-MRblockade interaction on the correlation between cortisol increase and amygdala connectivity with a large cluster covering both putamen and caudate (putamen: $\mathrm{p}_{\mathrm{SVC}}=0.003$, caudate: $\mathrm{p}_{\mathrm{SVC}}=0.004$, Figure 5 ). In line with our hypothesis, the association between cortisol increase and amygdala-striatal connectivity was significantly stronger in the stress group as compared to the control group, but only when the MR was available (putamen: $\mathrm{p}_{\mathrm{SVC}}=0.037$, caudate: $\mathrm{p}_{\mathrm{SvC}}=0.013$; $\mathrm{MR}$ blocked: no significant voxel). Thus, participants with stronger stress-induced cortisol increases showed not only enhanced amygdala activity, but also strengthened functional connectivity between amygdala and striatum. We did not find group differences in the relationship between cortisol increase and amygdala connectivity with other brain regions.

\section{DISCUSSION}

We set out to investigate how stress-related activation of the MR shifts the use of different spatial memory systems. In line with our hypothesis, our results provide first evidence that stress produces an increased dependence on spatial stimulusresponse memory in humans that is sensitive to blockade of the MR and involves an upregulation of amygdala activity and connectivity to the striatum.

The actions of cortisol are mediated by two receptor types, the MR and the glucocorticoid receptor (GR). Both MR and GR can induce rapid, non-genomic and slow, genomic effects mediated by receptors residing presumably at the membrane or in the cytoplasm, respectively (Joëls et al, 2012). An in-vitro study illustrated that MR-activation can rapidly enhance excitability of the amygdala (Karst et al, 2010). Other studies in rodents demonstrated that amygdala activation can in turn modulate the balance of spatial memory systems from hippocampal to striatal control over behavior (Packard and Teather, 1998; Packard and Wingard, 2004; Wingard and Packard, 2008). We translate these findings to humans by demonstrating a stress-induced MRdependent shift towards the striatum, orchestrated by increased amygdala activity and connectivity with the striatum, and especially present in those participants with high stress-induced cortisol responses. Considering the timing of these effects, ie within $45 \mathrm{~min}$ after stress onset, we tentatively conclude that these stress-induced effects likely result from rapid, non-genomic pathways, supposedly mediated by MRs located at or close to the plasma membrane.

The finding that MR-activation increases amygdala activity was never shown in humans, but was suggested by in-vitro experiments (Karst et al, 2010). These authors demonstrated rapidly enhanced excitability of amygdala neurons after corticosterone administration, which was driven by MRactivation, and subsequently sustained by GR-activation. This increase of amygdala activity was interpreted as 

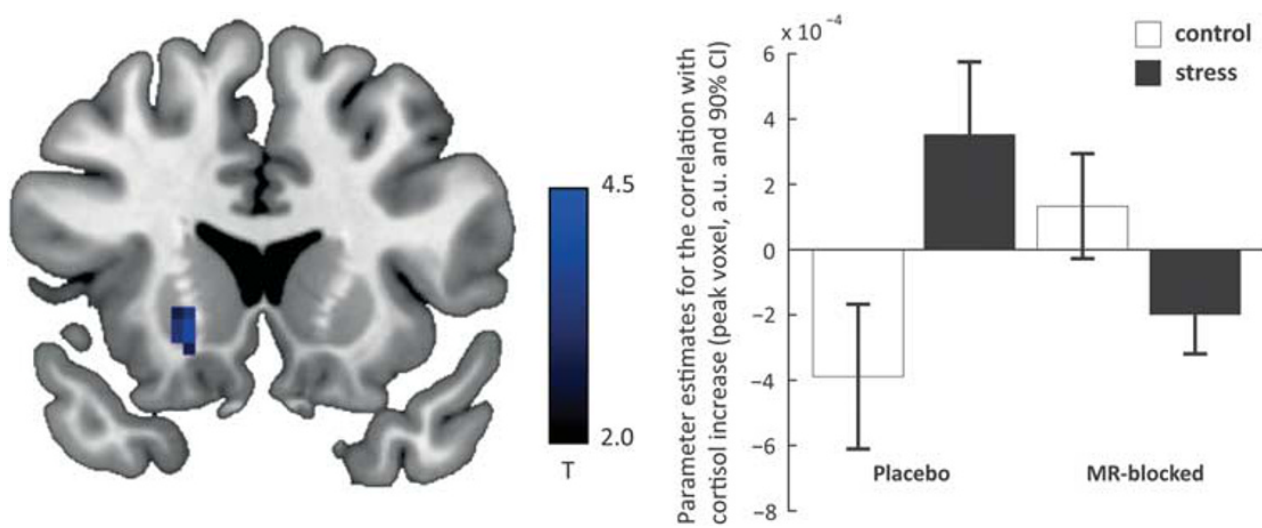

Figure 5 Left: The association between amygdala-striatal connectivity and cortisol increase measured as area under the curve was affected by stress and MR-blockade. For illustrative purposes, the image is thresholded at $p<0.005$, uncorrected, and masked for the region of interest (putamen). Right: For illustrative purposes only, we plot the parameter estimates for the correlation between amygdala-striatal activity and cortisol increase.

enabling enhanced memory encoding of stressful events. However, we show that MR-dependent amygdala upregulation may also lead to a qualitatively different memory trace by shifting processing towards another memory system. We also found that heightened cortisol levels induced by MR-blockade under non-stressful conditions were associated with enhanced amygdala activity. Given that the MR was blocked in these participants, this finding suggests that also the GR can enhance amygdala activation with moderate cortisol increases. One should keep in mind that spironolactone in itself caused an increase in cortisol levels, which was already present at least $25 \mathrm{~min}$ before stress onset. Therefore, in contrast to the rapid effect in the stress/placebo group, this up-regulation of amygdala activity in the control/ MR-blocked group cannot easily be attributed to rapid nongenomic effects, but might involve genomic GR-effects. Furthermore, when stress-induction was combined with moderately enhanced cortisol levels due to MR-blockade, we did not find an upregulation of amygdala activity, suggesting that the relation between GR activation and amygdala activity may be non-linear or depend on prior cortisol levels. To conclude, we interpret our findings as supporting an MR-dependent, stress-induced shift towards enhanced stimulus-response learning, which appears to be associated with an up-regulation of amygdala activity. However, future studies are needed to decipher the additional effects of GRactivation when the MR is blocked and cortisol levels are thus increased without an additional stress induction.

Recent studies on emotional face processing, fear learning, and probabilistic learning suggested that a stress-induced shift towards systems supporting habit-like behavior might arise from changes in amygdala connectivity with the hippocampus and the striatum (Schwabe et al, 2013a; Vogel et al, 2015b) or by affecting hippocampal activity (Vogel et al, 2015a). Beyond that, our current findings suggest that also amygdala activity is affected by MRactivation and might then orchestrate a stress-induced shift by changing connectivity to the striatum. Interestingly, we did not find such a stress-induced, MR-dependent enhancement of amygdala activity in the task directly following stressor onset (Vogel et al, 2015b). This might suggest that the stress-induced enhancement of amygdala activity might take about twenty minutes to arise which would be in line with in-vitro findings of enhanced amygdala excitability after corticosterone applications of $20 \mathrm{~min}$ (Karst et al, 2010) and a delayed rise of brain corticosterone levels after stress (Qian et al, 2011). Furthermore, the stress-induced increase in amygdala activity and connectivity may be transient as amygdala activity was found to be reduced one hour after hydrocortisone administration (Henckens et al, 2010) and no connectivity increase with the striatum could be detected one hour after stress onset (van Marle et al, 2010) or hydrocortisone administration (Henckens et al, 2012). These results highlight the striking time-dependency of stress effects on neural activity and memory (Henckens et al, 2010; Joëls et al, 2011; van Ast et al, 2013).

Another interesting aspect is that the heightened amygdala activity after stress was most pronounced during the feedback phase of the experiment when participants update and correct their memory. It appears that participants with higher stressinduced cortisol levels show stronger amygdala activity, especially during the updating of memories, biasing them towards stimulus-response learning. Previous experiments in rodents focused on the effects of a stress-induced shift between memory systems at encoding (Packard and Teather, 1998; Packard and Wingard, 2004; Wingard and Packard, 2008) or retrieval (Elliott and Packard, 2008). Our findings are the first to indicate that also the updating of already encoded (but not yet consolidated) information might be affected by a stress-induced shift in humans. Altogether, these findings suggest that multiple memory stages can be modulated by a stress-induced shift between memory systems.

Here, we used a translational approach in humans by testing a specific hypothesis concerning a stress-induced shift in memory systems which was derived from rodent studies. By employing a task which allowed the dissociation of 'cognitive' and 'habit' learning in behavior, we could determine the neural mechanism underlying a stressinduced shift in the balance of spatial memory systems: an MR-dependent up-regulation of amygdala activity and connectivity with the striatum. However, some caveats should be kept in mind. Spironolactone can also affect other receptors, for example progesterone receptors (Schane and Potts, 1978). As spironolactone also affects HPA axis regulation (de Kloet et al, 2005) it can change cortisol and corticotropin releasing factor concentrations, possibly 
contributing to the observed effects. Experiments with more specific drugs, possibly in rodents, might help in further specifying the role of the MR in the stress-induced shift. While a more specific MR-antagonist has been described (eplerenone), its effect on human cognition is still unclear and we chose for spironolactone in order to achieve comparability with earlier studies in humans (eg Cornelisse et al, 2011; Otte et al, 2007). Furthermore, also other neuromodulators may be involved in the stress-induced shift. For instance, it is conceivable that the MR might not directly act in the amygdala to change its activity and connectivity, but that these effects are mediated by dopamine in the ventral tegmental area (de Oliveira et al, 2014). The interplay between cortisol and other modulators is therefore a promising target for further research. Finally, it should be emphasized that the current study was carried out in male participants only due to practical limitations to our sample size and may not directly generalize to females (Ter Horst et al, 2013), although a stress-induced shift in females was demonstrated previously (Schwabe et al, 2013a).

Recent studies in other domains also support a stressinduced shift towards cognitively less demanding systems underlying behavior and learning (Arnsten, 2009; Otto et al, 2013; Vogel et al, 2015b). Beyond its effects on memory, stress also leads to more reflexive behavior (Porcelli and Delgado, 2009; Schwabe et al, 2011) and less strategic decisions being made. Altogether, these studies suggest a rapid stress-induced shift in neural processing, resulting in a dominance of less demanding systems across a broad range of cognitive domains. Our findings support the model proposed earlier (Schwabe et al, 2010) that the shift also affects spatial memory formation by inducing a dominance of habit-like stimulus-response learning. This shift towards the striatum might rescue memory performance under stress (Schwabe et al, 2010) but might also lead to strong memories which are hard to unlearn or update, potentially predisposing individuals (at least males) to develop psychopathology. Our data suggest that the stress-induced shift depends on the MR and may be prevented by the acute administration of MR-antagonists-or possibly other manipulations-dampening amygdala hyperactivity.

\section{FUNDING AND DISCLOSURE}

This study was supported by grants from the Netherlands Organization for Scientific Research (433-09-251 awarded to GF, MJ, MSO, and HJK; Vidi 452-12-009 awarded to CFD). GF and CFD are also supported by the European Research Council (GF: ERC-AiG NEUROSCHEMA R0001075; CFD: ERC-StG RECONTEXT 261177). The authors declare no conflict of interest.

\section{ACKNOWLEDGMENTS}

The authors thank Sabine Kooijman, Sanne Tops, Monique H.M. Timmer, Dirk Geurts, Niels ter Huurne, Atsuko Takashima, and Daphne Everaerd for their help acquiring data.

\section{REFERENCES}

Acheson DT, Gresack JE, Risbrough VB (2012). Hippocampal dysfunction effects on context memory: possible etiology for posttraumatic stress disorder. Neuropharmacology 62: 674-685.

Arnsten AFT (2009). Stress signalling pathways that impair prefrontal cortex structure and function. Nat Rev Neurosci 10: 410-422.

Cornelisse S, Joels M, Smeets T (2011). A randomized trial on mineralocorticoid receptor blockade in men: effects on stress responses, selective attention, and memory. Neuropsychopharmacology 36: 2720-2728.

de Kloet ER, Joels M, Holsboer F (2005). Stress and the brain: From adaptation to disease. Nat Rev Neurosci 6: 463-475.

de Oliveira AR, Reimer AE, Brandão ML (2014). Mineralocorticoid receptors in the ventral tegmental area regulate dopamine efflux in the basolateral amygdala during the expression of conditioned fear. Psychoneuroendocrinology 43: 114-125.

Doeller CF, Burgess N (2008a). Distinct error-correcting and incidental learning of location relative to landmarks and boundaries. Proc Natl Acad Sci 105: 5909-5914.

Doeller CF, King JA, Burgess N (2008b). Parallel striatal and hippocampal systems for landmarks and boundaries in spatial memory. Proc Natl Acad Sci USA 105: 5915-5920.

Elliott AE, Packard MG (2008). Intra-amygdala anxiogenic drug infusion prior to retrieval biases rats towards the use of habit memory. Neurobiol Learn Mem 90: 616-623.

Henckens M, van Wingen GA, Joels M, Fernandez G (2010). Time-dependent effects of corticosteroids on human amygdala processing. J Neurosci 30: 12725-12732.

Henckens MJAG, van Wingen GA, Joëls M, Fernández G (2012). Corticosteroid induced decoupling of the amygdala in men. Cereb Cortex 22: 2336-2345.

Herman CP, Polivy J (1975). Anxiety, restraint, and eating behavior. J Abnorm Psychol 84: 666-672.

Hermans EJ, Battaglia FP, Atsak P, de Voogd LD, Fernández G, Roozendaal B (2014). How the amygdala affects emotional memory by altering brain network properties. Neurobiol Learn Mem 37: 304-314.

Iaria G, Petrides M, Dagher A, Pike B, Bohbot VD (2003). Cognitive strategies dependent on the hippocampus and caudate nucleus in human navigation: variability and change with practice. J Neurosci 23: 5945-5952.

Joëls M, Baram TZ (2009). The neuro-symphony of stress. Nat Rev Neurosci 10: 459-466.

Joëls M, Fernandez G, Roozendaal B (2011). Stress and emotional memory: a matter of timing. Trends Cogn Sci 15: 280-288.

Joëls M, Sarabdjitsingh RA, Karst H (2012). Unraveling the time domains of corticosteroid hormone influences on brain activity: rapid, slow, and chronic modes. Pharmacol Rev 64: 901-938.

Karst H, Berger S, Erdmann G, Schutz G, Joels M (2010). Metaplasticity of amygdalar responses to the stress hormone corticosterone. Proc Natl Acad Sci USA 107: 14449-14454.

Kim JJ, Lee HJJ, Han JS, Packard MG (2001). Amygdala is critical for stress-induced modulation of hippocampal long-term potentiation and learning. J Neurosci 21: 5222-5228.

Morris RGM, Garrud P, Rawlins JNP, O'Keefe J (1982). Place navigation impaired in rats with hippocampal lesions. Nature 297: 681-683.

Otte C, Moritz S, Yassouridis A, Koop M, Madrischewski AM, Wiedemann $\mathrm{K}$ et al (2007). Blockade of the mineralocorticoid receptor in healthy men: Effects on experimentally induced panic symptoms, stress hormones, and cognition. Neuropsychopharmacology 32: 232-238.

Otto AR, Raio CM, Chiang A, Phelps EA, Daw ND (2013). Working-memory capacity protects model-based learning from stress. Proc Natl Acad Sci 110: 20941-20946. 
Packard MG, Teather LA (1998). Amygdala modulation of multiple memory systems: hippocampus and caudate-putamen. Neurobiol Learn Mem 69: 163-203.

Packard MG, Wingard JC (2004). Amygdala and 'emotional' modulation of the relative use of multiple memory systems. Neurobiol Learn Mem 82: 243-252.

Porcelli AJ, Delgado MR (2009). Acute stress modulates risk taking in financial decision making. Psychol Sci 20: 278-283.

Pruessner JC, Kirschbaum C, Meinlschmid G, Hellhammer DH (2003). Two formulas for computation of the area under the curve represent measures of total hormone concentration versus timedependent change. Psychoneuroendocrinology 28: 916-931.

Qian X, Droste SK, Gutièrrez-Mecinas M, Collins A, Kersanté F, JMHM Reul et al (2011). A rapid release of corticosteroid-binding globulin from the liver restrains the glucocorticoid hormone response to acute stress. Endocrinology 152: 3738-3748.

Schane HP, Potts GO (1978). Oral progestational activity of spironolactone. J Clin Endocrinol Metab 47: 691-694.

Schwabe L, Dalm S, Schachinger H, Oitzl MS (2008a). Chronic stress modulates the use of spatial and stimulus-response learning strategies in mice and man. Neurobiol Learn Mem 90: 495-503.

Schwabe L, Haddad L, Schachinger H (2008b). HPA axis activation by a socially evaluated cold-pressor test. Psychoneuroendocrinology 33: 890-895.

Schwabe L, Höffken O, Tegenthoff M, Wolf OT (2011). Preventing the stress-induced shift from goal-directed to habit action with a $\beta$-adrenergic antagonist. J Neurosci 31: 17317-17325.

Schwabe L, Oitzl MS, Philippsen C, Richter S, Bohringer A, Wippich W et al (2007). Stress modulates the use of spatial versus stimulus-response learning strategies in humans. Learn Mem 14: 109-116.

Schwabe L, Schachinger H, de Kloet ER, Oitzl MS (2010). Corticosteroids operate as a switch between memory systems. J Cogn Neurosci 22: 1362-1372.

Schwabe L, Tegenthoff M, Höffken O, Wolf OT (2013a). Mineralocorticoid receptor blockade prevents stress-induced modulation of multiple memory systems in the human brain. Biol Psychiatry 74: 801-808.
Schwabe L, Wolf OT (2013b). Stress and multiple memory systems: from 'thinking' to 'doing'. Trends Cogn Sci 17: 60-68.

Taube JS (1998). Head direction cells and the neurophysiological basis for a sense of direction. Prog Neurobiol 55: 225-256.

Ter Horst JP, Kentrop J, Arp M, Hubens CJ, de Kloet ER, Oitzl MS (2013). Spatial learning of female mice: a role of the mineralocorticoid receptor during stress and the estrous cycle. Front Behav Neurosci 7: 56.

Tzourio-Mazoyer N, Landeau B, Papathanassiou D, Crivello F, Etard O, Delcroix N et al (2002). Automated anatomical labeling of activations in SPM using a macroscopic anatomical parcellation of the MNI MRI single-subject brain. Neuroimage 15: 273-289.

van Ast VA, Cornelisse S, Meeter M, Joels M, Kindt M (2013). Time-dependent effects of cortisol on the contextualization of emotional memories. Biol Psychiatry 74: 809-816.

van Marle HJF, Hermans EJ, Qin SZ, Fernandez G (2010). Enhanced resting-state connectivity of amygdala in the immediate aftermath of acute psychological stress. Neuroimage 53: 348-354.

Vogel S, Klumpers F, Kroes MC, Oplaat KT, Krugers HJ, Oitzl MS et al (2015a). A stress-induced shift from trace to delay conditioning depends on the mineralocorticoid receptor. Biol Psychiatry 78: 830-839.

Vogel S, Klumpers F, Krugers HJ, Fang Z, Oplaat KT, Oitzl MS et al (2015b). Blocking the mineralocorticoid receptor in humans prevents the stress-induced enhancement of centromedial amygdala connectivity with the dorsal striatum. Neuropsychopharmacology 40: 947-956.

Weiss F, Ciccocioppo R, Parsons LH, Katner S, Liu XIU, Zorrilla EP et al (2001). Compulsive drug-seeking behavior and relapse. Ann N Y Acad Sci 937: 1-26.

Wilton LA, Baird AL, Muir JL, Honey RC, Aggleton JP (2001). Loss of the thalamic nuclei for 'head direction' impairs performance on spatial memory tasks in rats. Behav Neurosci 115 : 861-869.

Wingard JC, Packard MG (2008). The amygdala and emotional modulation of competition between cognitive and habit memory. Behav Brain Res 193: 126-131.

Supplementary Information accompanies the paper on the Neuropsychopharmacology website (http://www.nature.com/npp) 